\title{
Grafoscopio: un prototipo/hipótesis para indagar sobre cómo cambiamos los artefactos digitales que nos cambian*
}

\author{
Graphoscope: A Prototype/Hypothesis to Enquire into How We Change the Digital Devices Changing Us \\ Grafoscópio: um protótipo/hipótese para indagar sobre como mudamos os artefatos digitais que nos mudam
}

\author{
Offray Vladimir Luna Cárdenasa ${ }^{\text {a }}$ \\ Universidad de Caldas, Colombia \\ offray.luna@mutabit.com \\ ORCID: https://orcid.org/0000-0002-4640-6984
}

DOI: https://doi.org/10.11144/Javeriana.syp39-77.gpic

Recibido: 20 Abril 2020

Aceptado: 20 Agosto 2020

Publicado: 30 Diciembre 2020

\section{Resumen:}

Este artículo da cuenta de un prototipo digital que indaga por la modificación recíproca entre comunidades de base y artefactos digitales a la vez que encarna hipótesis al respecto de cómo potenciar dicha modificación. Para ello, muestra las conexiones de dicho artefacto con movimientos de ciencia abierta, ciudadana y de garaje, de objetos activistas y de investigación y de la tecnopolítica del software libre. También considera elementos de diseño tenidos en cuenta para la creación del prototipo (autorreferencialidad y boostraping), así como metodológicos desde las apuestas del software como artesanía, como hipótesis y como manera de vincularse y conformar comunidades de práctica.

Palabras clave: investigación, creación, ciencia ciudadana, artefactos digitales, investigación basada en diseño, narrativas y visualización de datos.

\begin{abstract}
:
This article deals with a digital prototype in order to inquire into the reciprocal modification between grassroots communities and digital devices that provides a hypothesis on how this modification can be leveraged. To do so, this work shows the relationships between this device and the movements of open-, citizen-, garage-science, activist and research objects and free software technopolitics. It also examines the design elements taken into account when creating the prototype (self-reference and boostraping) as well as the methodological elements assuming the software as a handicraft; all this is taken as a hypothesis and as a way of forming and engaging with practice communities.
\end{abstract}

Keywords: research, creation, citizen science, digital devices, design-based research, narratives and data displaying.

\section{Resumo:}

Este artigo dá conta de um protótipo digital que investiga a alteração recíproca entre comunidades de base e artefatos digitais ao mesmo tempo em que incorpora hipóteses sobre como potenciar tal alteração. Para isso, mostra as conexões de esse artefato com movimentos de ciência aberta, cidadã e de garagem, de objetos ativistas e de pesquisa e da tecnopolítica do software libre. Mesmo considera elementos de design levados em consideração para a criação do protótipo (autorreferencialidade e boostraping), bem como metodológicos desde as apostas do software como artesanato, hipótese e maneira de se vincular e formar comunidades de prática.

Palavras-chave: pesquisa-criação, ciência cidadã, artefatos digitais, pesquisa baseada em design, narrativas e visualização de dados.

\author{
Todo lo que puedas *terminar ${ }^{*}$ encarnará su propia critica \\ Perfection \& Feedback Loops \\ or: why worse is better \\ Markus Denker (2016)
}

Notas de autor

\footnotetext{
a Autor de correspondencia. Correo electrónico: offray.luna@mutabit.com
} 


\section{Introducción}

La Comunicación para el Desarrollo o para el Cambio Social (CDCS) indaga por la apropiación ciudadana de medios alternativos y los llamados "nuevos medios" apoyados en Tecnologías de la Información y la Comunicación (TIC) (Carretero, 2019). Dicha averiguación debe dar cuenta también de la transición hacia un conjunto de medios de comunicación algorítmicos con dos características diferenciales: 1) pasan desde broadcast, con un emisor centralizado y muchos receptores, como la radio y la televisión (en sus variantes privadas, estatales y comunitarias), hacia uno multicast de muchos emisores y receptores, como el de las redes sociales y 2) donde el procesamiento algorítmico embebe de agencia a las plataformas, perfila constantemente cada internauta para influirle, personalizar lo que percibe, configurando a su alrededor las llamadas burbujas de información (Pariser, 2012). Una vez configurado el mensaje, los medios y dispositivos tradicionales lo emitían y recibían, mientras que los digitales, además lo procesan y cambian a medida, agrupando usuarios y embebiendo intereses, sesgos y agendas de programadores y dueños de las plataformas (O’Neil, 2016), más allá de lo que era posible con los medios tradicionales.

En este contexto, la pregunta por lo algorítmico y lo informático cobra relevancia en los estudios en comunicación y las publicaciones investigativas asociadas, dando cuenta de campos emergentes (software studies, critical code studies, sociología digital) que requieren diálogos articulados con experticias y lenguajes algorítmicos, a la vez que enfrentan el desafío de moverse en espacios liminales, donde las tensiones y brechas entre los distintos saberes y lenguajes (lo comunicativo, lo comunitario, lo activista, lo algorítmico, el software, la publicación indexada o no) se hacen evidentes y se avisoran los puentes.

El presente artículo encarna tales dificultades y pretende aportar en estos diálogos puente, desde la perspectiva de la investigación en la transdiciplina del diseño. Para ello, muestra los resultados de la pregunta por “¿cómo cambiamos los artefactos digitales que nos cambian?", es decir, la indagación por el cambio recíproco entre comunidades y herramientas digitales. Acá se examina la apropiación comunitaria, no tanto de los medios (antiguos o nuevos), sino de los metamedios, en otras palabras, aquellos medios con los cuales construimos e integramos otros medios y mediaciones (en este caso, texto, código, datos, visualizaciones y sistemas de autopublicación), debido a la manera como esta integración particular explicita el carácter algorítmico de los medios digitales y abre caminos a su re-apropiación y deconstrucción.

Además de vincular en lo teórico las tradiciones del diseño basado en investigación y ser un eslabón en la larga cadena de apropiación de medios de la CDCS, la investigación por la apropiación comunitaria de los metamedios tiene correlatos prácticos en las formas en que comunidades de base y ciudadanías (re)configuran tecnologías para amplificar sus voces, como se mostrará acá.

La primera parte del texto presenta una manera particular de investigar en diseño desde la construcción de un prototipo digital y la articulación de comunidades alrededor. También muestra las apuestas metodológicas y políticas de traer, vía el prototipo, prácticas de mundos posibles al presente al develar el contexto hacker donde dichos acercamientos ocurrieron. La segunda parte indaga sobre el prototipo mismo que para esta investigación se construyó, Grafoscopio, ubicándolo en diálogo con movimientos de ciencia ciudadana y de garaje, ciudadanías digitales, activismo e investigación y publicación reproducible. Además, da cuenta de un conjunto particular de propiedades más abstractas en el diseño del mismo (por un lado, boostrapping y autoreferencialidad y por otro bifuración y recombinación), que pueden ser útiles a la hora de considerar otras maneras de (re)apropiación de (meta)medios algorítmicos. La última parte ofrece una serie de conclusiones y muestra lugares de investigación a futuro. 


\section{Metodologías de investigación/creación}

El diseño como transdiciplina y saber en red (Jonas y Meyer-Veden, 2004; Saikaly, 2005) puede ayudar a explorar saberes complejos y lugares liminales, como el ya referido a propósito de una mayor relevancia de lo algorítmico en los medios de comunicación y la (re)apropiación comunitaria de los metamedios digitales. Y, para las epistemologías en diseño, el prototipo es una pieza clave, pues sirve como laboratorio y como medio (Saikaly, 2005), ya que se usa tanto para experimentar sobre las hipótesis plausibles que la investigación en diseño busca develar como para comunicarlas.

Dado que prototipar es indagar por lo posible, eligiendo y encarnando alguna de las posibilidades para traerla al presente, inevitablemente, al prototipar se hacen una serie de apuestas políticas desde espacios específicos (Luna Cárdenas, 2018). Es decir, que debido al carácter encarnado de la investigación en diseño cuando esta prototipa (algo, para y con alguien, en algún contexto), no se debería acudir a la neutralidad como su lugar de rigor académico, sino a la transparencia. Esta investigación asume el carácter del investigador como sujeto político, que no investiga sobre las comunidades, sino desde ellas y las entiende como co-productoras del conocimiento que este lleva a la academia en los limitados formatos que esta visibiliza, valora y espera. Pero esta investigación entiende el conocimiento y su circulación como no neutros y al reconocerlo se intenta enmarcar dentro de las tradiciones de academia activista (Derickson y Routledge, 2015), más cercana, comprometida, sensible y resonante a las comunidades desde las que co-investiga, más consciente de las dinámicas de poder entre investigadores y comunidades, así como de los peligros del llamado extractivismo cognitivo.

Por lo anterior, los apartes de esta sección procuran transparentar las aproximaciones desde las cuales se construyó Grafoscopio, el prototipo de esta investigación, estableciendo metodologías de investigación en diseño que consideran a software (y sus prototipos) como hipótesis y luego considerando una aproximación al software como labor artesanal, a diferencia de los enfoques ingenieriles con los cuales se suele asociar comúnmente esta práctica creativa y cómo se asume el espacio hacker o hackerspace), HackBo, el contexto específico donde Grafoscopio encarnó e iteró, como un espacio de repolitización de la tecnología.

\section{El software como hipótesis y como experiencia de aprendizaje comunitario}

Un aspecto central de la metodología seguida en esta investigación está explicado en Software as Hypothesis: Research-Based Design Methodology (Leinonen et al., 2008). Se asume, precisamente, a los prototipos de software como hipótesis y se ponen a circular en contextos colectivos y comunitarios, con permanentes ciclos de realimentación durante todo el proceso. Esta metodología de diseño está caracterizada por las fases que se presentan en la Figura 1. 


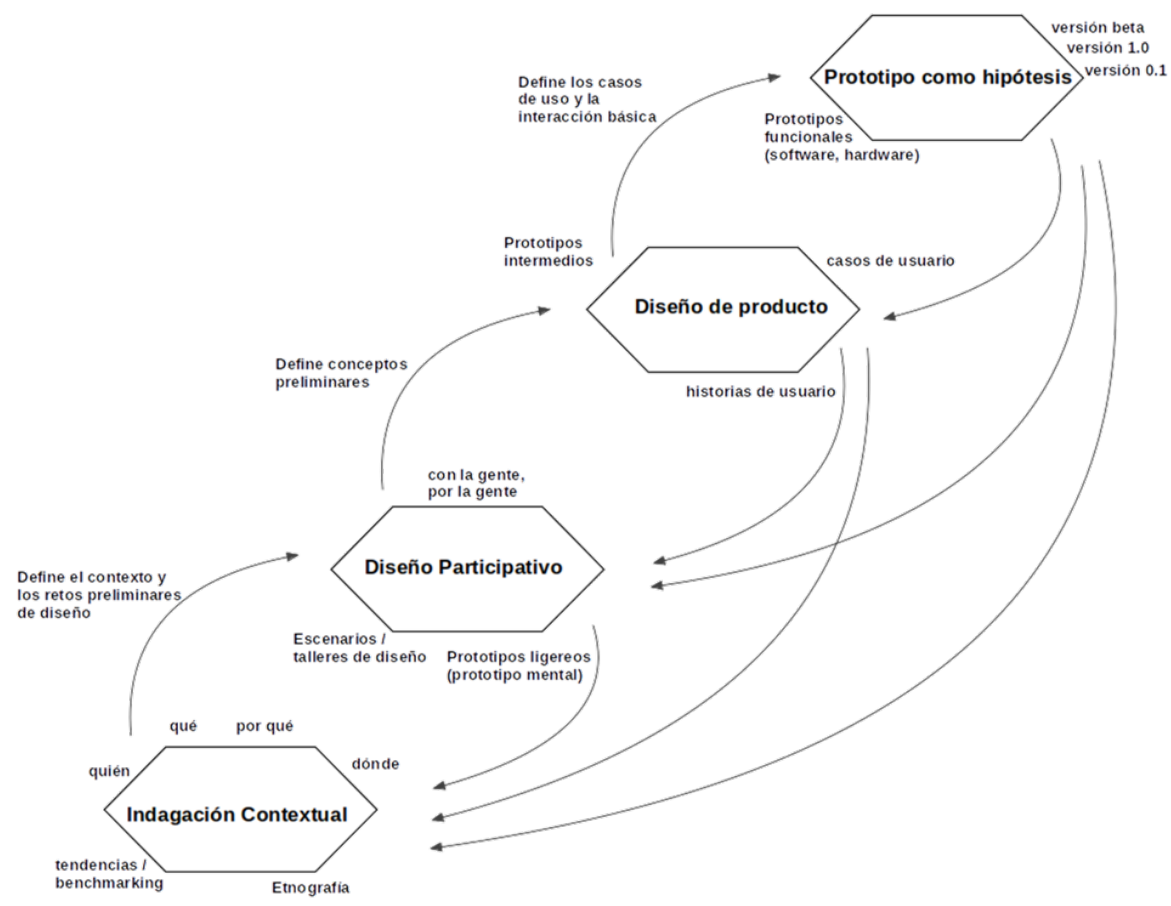

FIGURA 1.

Dinámica de diseño para la modificación recíproca entre artefactos y comunidades.

Fuente: Adaptada de Leinonen et al. (2008, p. 31).

1. Indagación contextual: En esta, se indaga por el contexto sociocultural en el cual ocurre el diseño. Se pregunta por el quién, qué, el por qué y dónde de la investigación, para lo cual se pueden usar técnicas etnográficas rápidas. En la medida en que se hace el trabajo de campo, se realizan indagaciones focalizadas sobre la literatura y pruebas de desempeño (benchmarking) sobre soluciones preexistentes o posibles.

2. Diseño participativo: En esta fase, se trabaja con los incumbentes (stakeholders) a través de talleres y prototipos ligeros o mentales del tipo "qué tal si...”. Acá los prototipos como tal no existen, sino que se formulan hipótesis sobre cuáles podrían ser los productos y prototipos que den cuenta de las necesidades del contexto encontradas en la fase previa.

3. Diseño de producto: En esta fase, se crean prototipos tempranos por parte del grupo del proyecto y se mantiene distancia de los stakeholders, pues la discusión suele ser de carácter altamente técnico usando lenguajes especializados para la misma.

4. Prototipo como hipótesis: Acá se ponen a circular los prototipos para ser validados o no por los stakeholders. Su carácter de hipótesis es lo que permite su constante revaluación dentro de los límites del proyecto.

Como se dijo, estas fases tienen ciclos de realimentación permanentes, y se puede empezar en cualquiera de las fases para volver a las anteriores o ir a las siguientes. A continuación, se presentará el contexto donde este prototipado ocurre, en primera instancia y se aplazará la presentación del prototipo, y cómo este se fue iterando y socializando en dicho contexto, mientras que la sección de principios de diseño dará cuenta de las realimentaciones expuestas en la metodología previa. 


\section{HackBo: un hackerspace en Bogotá}

Esta investigación ocurrió principalmente en HackBo (HackBo, s. f.), un hackerspace en Bogotá. Los hackerspace son espacios de aprendizaje disfrazados donde, al establecerse relaciones cotidianas con la tecnología, los participantes de dichos espacios se hacen hackers sin darse cuenta (Schrock, 2014). Schrock (2018) preserva la invitación de Coleman (2013) y Wark (2004) a mantener la definición de lo hacker como abierta y multisituada, e indica que los hackers son ordinarios, en el sentido de que no es esa figura mítica, quien gracias a una comprensión especial de la tecnología la domina, sino que en una relación dialéctica con ella es la tecnología la que hace al hacker en actos cotidianos: al soldar, programar y "cacharrear" con mucha frecuencia. Los hackerspaces son lugares para configurar y experimentar esa relación en el diario vivir. Esta noción de lugar de aprendizaje disfrazado resuena con la de comunidad de práctica (Wenger, 1999), pues en el hackerspace encarnan, transitan, se socializan y reconfiguran repertorios simbólicos y materiales particulares de la cultura hacker. HackBo es también un ejemplo de los lugares citados por Manzini y Meroni (2013) para innovación social emergente, donde se codiseñan alternativas para habitar el mundo, en la medida en que quienes lo ocupan no se conciben ni vivencian a sí mismos desde las narrativas hegemónicas asociadas a la tecnología, el co-working o incluso lo hacker. HackBo es así un espacio polisémico y plurárquico que tiene lecturas y posibilidades distintas para quienes lo construyen y habitan. Como todo espacio, está regido por tres fuerzas, según Isin y Ruppert (2015) performativas, legales e imaginarias, que se refieren, respectivamente, a lo que ocurre en el espacio, a su condición legal y a lo que nos imaginamos que puede ser y ocurrir. En un lugar así, ¿qué actividades constituyen el hackear dentro de un hackerspace? Debido a este carácter abierto de lo hacker presentado acá, más que acotar una definición, en esta investigación se suscribe a una en particular del fenómeno hacker y lo asume desde la repolitización de la tecnología (en particular) la digital. En la acepción de este trabajo, no todo es hackear, con lo cual no se busca agotar el fenómeno, pero sí articularlo con los alfabetismos digitales que, mediados por código y datos, se ubican en clave de derechos. HackBo, el hackerspace contexto de esta investigación, es entonces un espacio polisémico que ofrece múltiples lugares de vinculación y dado que prototipar es elegir, las elecciones hechas en ese contexto se afilian a la idea de lo hacker como la repotilización de la tecnología para deconstruir relaciones de poder y explorar maneras de ejercer lo ciudadano, particularmente desde las nociones de hacker cívico (Schrock, 2016), hacktivismo, ciudadanías digitales de Isin y Ruppert (2015), donde fuerzas enactivas, legales e imaginativas permiten pensarnos como sujetos (y comunidades) de poder y al poder, en particular cuando re-apropiamos y conectamos datos, documentación, visualizaciones y publicaciones para ejercer y re-imaginar derechos.

El código, en relación con los metamedios, da cuenta de las maneras en que decimos cosas a través de actos digitales (Isin y Ruppert, 2015) e infraestructuras en las que el código cristaliza dicha repotilización de la técnología y que re-ensambla las fuerzas performativas, imaginativas y legales de los espacios cívicos, en particular de los hackerspaces.

\section{Prototipos como formas de vincularse a comunidades y conformarlas}

El desarrollo de software en esta investigación también es visto como un acto de enculturación, desde la perspectiva de Wenger (1999). Se trata de ir adquiriendo los repertorios simbólicos y materiales compartidos por una comunidad de práctica. "Prototipar" es, además, explorar y apropiar ese repertorio en la medida en que se interactúa con comunidades particulares, que en el caso del software libre, se articulan alrededor de los artefactos que usan y lo que estos posibilitan. Es por ello habitual que las comunidades de software libre reciban el nombre de tales artefactos: Debian o $\mathrm{Gnu} /$ Linux denominan tanto a sistemas operativos como a comunidades, IPython/Jupyter o Pharo denotan tanto lenguajes y entornos de programación como 
comunidades. De modo similar, Grafoscopio describe tanto a un artefacto de software, como la comunidad articulada por las indagaciones que el mismo cristaliza.

La pregunta por la modificación recíproca entre artefactos digitales y comunidades llevó a un acto de apropiación dentro de unas comunidades de práctica de software libre - las de Pharo (2017), Fossil (Luna Cárdenas, 2014), entre otras - que permitió explorar y expresar búsquedas sobre lo escritural, sobre las relaciones entre artefactos digitales, conocimiento, ciudadanía y publicación, sobre los datos y la forma de contar historias, así como sobre las infraestructuras que permiten participar o no en dichas posibilidades. Estas exploraciones ocurrieron primero a nivel personal, apropiando las materialidades y rituales propios de dichas comunidades, y luego se pensaron en maneras de tejer puentes, de doble vía, entre preocupaciones locales que podrían ser expresadas por artefactos como Grasfoscopio, y formas comunitarias de hacer y aprender. Los prototipos exploraron y comunicaron maneras de pensar el software desde lo artesanal y su creación como un acto de enculturación comunitaria, así como maneras de expresar compresiones y materialidades progresivamente más detalladas, que a su vez permitieron construir los prototipos/hipótesis y desde ellos participar de otra maneras de publicación y presencia, tanto localmente, en forma de tutoriales, entradas de blog, wikis, talleres, hackatones y otros prototipos, como en publicaciones indexadas, manuales y libros (en inglés y español), además de participación en eventos internacionales. La investigación entra a través de estas materialidades, prácticas y participaciones en diálogos con comunidades preexistentes de software libre y libre cultura. Pero también acompaña y propicia su conformación y transformación hacia otros espacios (HackBo, la comunidad de Grafoscopio), que continúan una vez la investigación formal se ha acabado y los objetos académicos convencionales de la misma se han publicado y socializado. Es una investigación que interpela a las comunidades, retornando y continuando principalmente en ella (a través de prototipos y talleres), aunque estableciendo vínculos con la academia (principalmente en tesis doctorales y artículos indexados).

\section{Una aproximación artesanal y sus alcances}

El desarrollo principal de Grafoscopio ocurrió intensamente durante tres años y medio (2014 a 2018) y se ha continuado desarrollando después, de modos más pausados, debido a los usos actuales en los contextos locales e internacionales. Como se dijo, el desarrollo de este software no es cercano a prácticas ingenieriles tradicionales, sino que se enmarca en la idea de aprendizaje como un acto de enculturación en una comunidad de práctica (Wenger, 1999) y la acepción del software como artesanía (Blackwell y Aaron, 2015), de la que nos ocuparemos acá.

Desde dicha aproximación, el software embebe y encarna conocimiento crítico de su(s) autor(es) y es un "material recalcitrante" (Blackwell y Aaron, 2015), con el que dialogamos y que nos permite investigar mediante la práctica reflexiva. Dicha materialidad del software permitiría establecer diálogos y prioridades, dejando que el material nos guíe, específicamente en la relación entre tales materialidades y las comunidades alrededor de ellas. Según tales autores (pp. 2-3),

Las herramientas prácticas artesanales han 'evolucionado' para adecuarse a la mano experta a través de generaciones de uso de hecho, 'co-evolucionaron' porque el entrenamiento artesanal procede junto con las prácticas reflexivas de hacer y adaptar las herramientas propias. Podría entonces esperarse que la artesanía del software estuviera parcialmente 'encarnada' en las herramientas de programación que codifican las prácticas expertas evolucionadas, tales como el prototipado, la modelación y el refactoring.

$[\ldots]$

La comprensión del software como materialidad inicialmente parece contraintuitiva, por el hecho de que el software es por supuesto inmaterial. Sin embargo, podemos usar la comprensión de la materialidad en la interacción (Gros et al., 2013) para observar que el código es usualmente un medio recalcitrante, que ofrece resistencia a la manipulación por el programador, en la misma manera que lo hacen los materiales mediales de la práctica artística. 


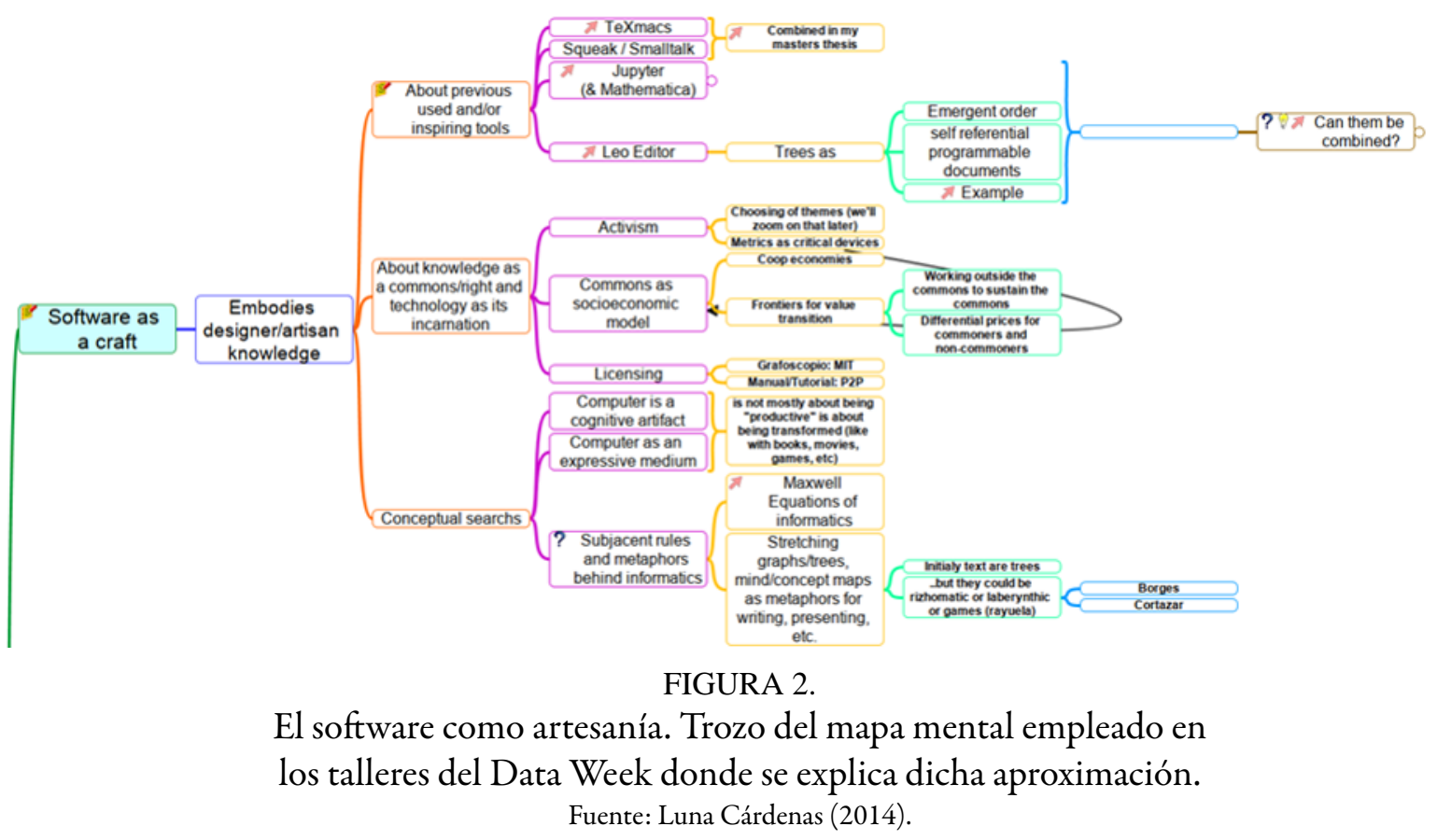

Fue así como durante el desarrollo de Grafoscopio se tuvieron momentos frenéticos con exploración intensiva de las posibilidades y prioridades (particularmente al comienzo) y también ritmos más sosegados, logrados gracias a la interacción con la naciente comunidad de Grafoscopio. El prototipo, avanzó como decimos en dicha comunidad, "sin prisa, pero sin pausa" y no buscó una experiencia absolutamente fluida y limpia. En cambio, se entregó un prototipo funcional básico, que satisficiera las condiciones mínimas, para que fuera la interacción entre prototipo y comunidad la que dictara las prioridades siguientes, en concordancia del prototipo como hipótesis y los ciclos de realimentación de la investigación en diseño, teorizados por Leinonen et al. (2008): indagación contextual, diseño participativo, diseño de producto y software como hipótesis.

Grafoscopio fue el primer prototipo desarrollado, por el autor, en la variante Pharo de Smalltalk y de hecho la primera aplicación de usuario escrita por este, que brindaba desafíos distintos de las experiencias de modelado algorítmico previas. Las demandas nuevas requerían una aproximación que fuera ágil. Y debido a la inexperiencia y desconocimiento sobre metodologías más formales de desarrollo de software, se procedió de una manera amateur, aprendiendo durante la marcha y aumentando la formalidad en la medida que fuera necesario. Conforme se iba aprendiendo, algunas partes del prototipo eran rehechas, en un proceso que en desarrollo de software se conoce como refactoring. Allí Pharo mostró otra de sus ventajas, pues no cobraba caro las decisiones tempranas propias del carácter de programador novato, sino que le permitía a un futuro programador más experto, revaluar las decisiones que había tomado su versión más novata y rehacerlas sin mayor dificultad $^{1}$. La parte escritural provee una interfaz básica y se apeló al lenguaje de etiquetamiento ligero Markdown para los elementos de formato (Gruber, 2004), confiando en que esa interfaz sencilla, unida al valor diferencial del prototipo digital como otra manera de organizar el texto y vincularlo con visualizaciones, fuera suficientemente llamativa para los miembros de la comunidad que quisieran continuar usando el software. Los Lenguajes de Domino Específico (DSL, por sus siglas en inglés) para el procesamiento de texto y la visualización de datos también fueron surgiendo de manera emergente de acuerdo a la necesidad y han continuado afinándose en eventos locales e internacionales en los que son requeridos. La escritura, el desarrollo y compresión explícita de los DSL es parte de las intenciones de uso detrás de Grafoscopio, por lo que no se espera proveer metáforas visuales que los oculten o hagan que los usuarios no se enfrenten a este aspecto del código. Sin embargo, si se espera mejorar la Interfaz Gráfica de Usuario (GUI, por sus siglas en inglés), de modo que el trabajo con toda la funcionalidad de Grafoscopio, incluidos los DSL sea más fluida, siguiendo con la idea de poner a circular e iterar prototipos mínimos y funcionales desde los cuales detonar dichas experiencias futuras y revaluar las elecciones de diseño del pasado. 
Lo anterior da cuenta parcial de cómo la interacción desde una perspectiva artesanal con el código y su circulación en comunidad fue orientando el quehacer y los aprendizajes futuros, llenando o dejando huecos en el saber, dependiendo de su utilidad en los contextos circundantes. De la conexión con dichos contextos se ocupa la siguiente sección.

\section{Grafoscopio y su lugar en las alteridades de la investigación}

\section{¿Qué es Grafoscopio?}

Grafoscopio es una herramienta amoldable para publicación e investigación reproducible y narrativas y visualización ágiles de datos (Luna Cárdenas, 2017a), que permite mezclar prosa, código, datos y gráficos y exportarlos a formatos web (HTML) e impresos (PDF).

Grafoscopio realiza varios aportes en el contexto de indagar por la modificación recíproca entre comunidades y artefactos digitales, desde perspectivas críticas: es altamente cambiable, debido a estar realizado en el metasistema de live coding o programación en vivo Pharo (un sistema hecho en sí mismo); posee una metáfora ${ }^{2}$ uniforme para trabajar a lo largo del entorno (conocida técnicamente como un enfoque objetual puro), lo cual facilita su aprendizaje y modificación; tiene una perspectiva latinoamericana, desde la apuesta por lo que se denominó como "infraestructuras de bolsillo" (sencillas, extensibles, amoldables, autocontenidas y que funcionan bien en y fuera de línea). Además, realiza un puente entre el mundo impreso y el mundo computacional, pues al combinar prosa, código, texto, datos y visualizaciones en investigaciones y publicaciones reproducibles, con una fuerte apuesta por la llamada tecnología cívica, permite un ingreso de más saberes y sujetos, además de los habituales desarrolladores de software o los científicos de la computación. Las interfaces gráficas de usuario o GUI, funcionan como metáforas, conectando dos sistemas simbólicos distintos. Así en lugar de manipular voltajes en procesadores masivamente integrados, los usuarios de computadores manipulan íconos, punteros, símbolos y ventanas que se organizan bajo una experiencia particular regida por la(s) metáfora(s) que los unifican (la tabla en la hoja de cálculo, la diapositiva en el programa de presentaciones, etc.).

Desde la GUI, Grafoscopio se aparta de la metáfora escritural de la página en blanco, que se llena renglón a renglón y la barra de herramientas saturada que explicitan los procesadores de palabra populares (MS Word, Libre Office Writer o Apple Pages) y su interfaz WYSIWYG ("What you see is what you get", por "lo que ves es lo que obtienes"). En su lugar, presenta la metáfora del documento en el computador como una estructura arbórea ${ }^{3}$, que da a las palabras e ideas secuencia y jerarquía, mostrando en simultánea distintas partes del documento, y permitiendo ocuparse de las mismas en la medida que el texto asociado a ellas va ocurriendo (Figura 3). Las ramas en ese árbol (conocidas como nodos) reflejan distintos niveles de granularidad y contenencia del documento, según emergen y se reorganizan: capítulos, secciones, subsecciones e incluso ideas o párrafos. Los nodos son esencialmente de dos tipos, de prosa y de código, y estos últimos pueden describir y ejecutar trozos del documento para acceder a bases de datos, realizar visualizaciones o cambiar la manera en que el documento es exportado. 


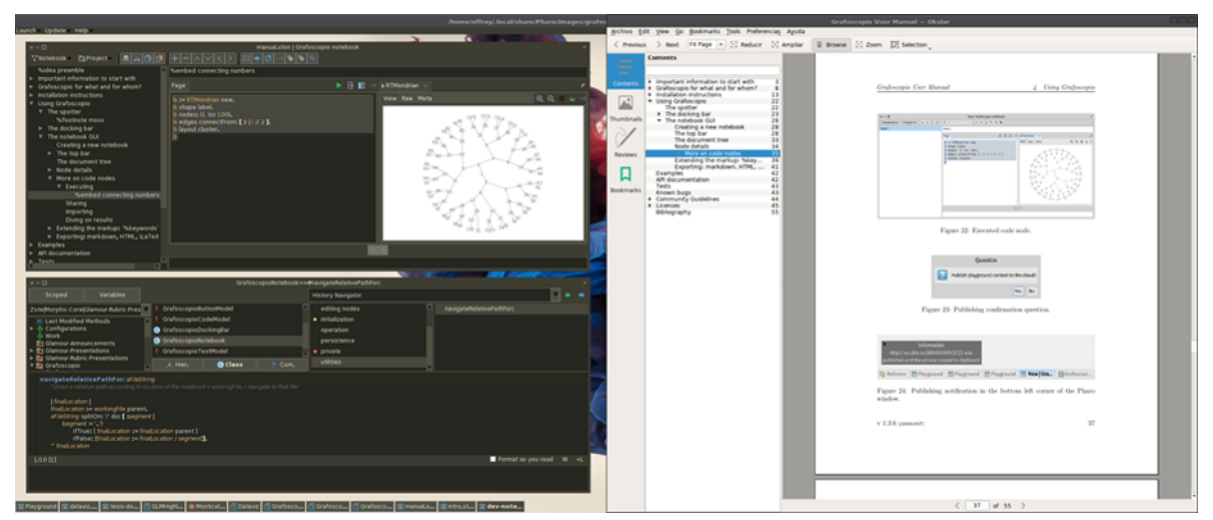

FIGURA 3.

Grafoscopio, interfaz y exportación de documentos.

Izquierda (arriba): El documento arbóreo interactivo con un nodo de código que produce una gráfica; (abajo): el navegador de código para extender y modificar el entorno en caliente. Derecha: El documento exportado como PDF, donde el árbol se refleja en la tabla de contenido. Fuente: Luna Cárdenas (2014).

Dentro de las múltiples intensiones de Grafoscopio, hay dos entrelazadas, que vale la pena resaltar:

- Explorar formas de escribir y publicar diferentes, que permitan amplificar las voces de las comunidades de base, usando maneras de argumentar desde los datos y las visualizaciones. En particular, interesan los argumentos que se dan en las interacciones entre dichas comunidades y entidades estatales, pero también aquellos en referencia a otros campos que configuran las llamadas ciudadanías digitales (Isin y Ruppert, 2015), incluyendo hackatones civiles para la gestión de lo público, talleres de aprendizaje entre pares de código, desarrollo de tecnologías cívicas, autopublicación y gestión de datos personales, que recuperamos de la permanente expropiación realizada por las redes sociales populares —ejemplos de estas nuevas prácticas están descritos en Luna Cárdenas (2018) -

- Visibilizar los múltiples objetos de investigación, de los cuales la academia suele no dar cuenta, debido a las prácticas de validación de saberes que privilegian excesivamente lo escrito y la publicación indexada ${ }^{4}$, mediante la vinculación al texto de repositorios de código fuente, bases de datos y bibliográficas, algoritmos, entre otros. Estas dos búsquedas se enlazan al construir nuevas metáforas escriturales, que a su vez permitiesen adquirir nuevos alfabetismos sobre lectoescritura, mediada por código, datos y visualización. Esto a su vez permitiría extender, re y co-construir el artefacto original, cambiando asi el artefacto que nos cambia. A continuación, se explicitarán los lugares de alternos de investigación en los que se enmarca Grafoscopio y cómo este dialoga en particular con dichos lugares.

\section{Investigaciones y ciencias otras, objetos de investigación reproducibles y activistas}

Distintas iniciativas, colectivas e individuales están deconstruyendo y reconfigurando las prácticas con las cuales se apropian, producen y comunican saberes. Tales iniciativas agrupan bajo distintas denominaciones, como investigación y ciencia abiertas, ciencia de garaje, research object, activist object (se hará referencia a ellas de modo colectivo con la sigla ICACG), complementado y en muchas ocasiones contrastando críticamente las maneras y lugares hegemónicos desde los que se realizan las labores de apropiación, producción y comunicación de saberes al interior de la academia y se repiensan los pactos entre esta y la ciudadanía. Podemos, entonces, imaginar tránsitos de doble vía de saberes y comunicación, que también van desde la 
ciudadanía hacia las instituciones científicas para revertir esa lógica, donde las comunidades son vistas como simples "objetos de estudio" y se convierten en "sujetos estudiosos", y donde tampoco son "usuarios finales" de lo que la ciencia produce y es mediado por el mercado y entregado a ciudadanos y comunidades vía la tecnología. Los colectivos e individuos, en su carácter de académicos vinculados a las instituciones, como ciudadanos fuera de ellas, o en algún lugar intermedio, están pensando en maneras distintas de comunicar las respuestas que saberes académicos tradicionalmente se han hecho, de colocar nuevas preguntas en la intersección entre saberes o de abordar de manera más horizontal y participativa la construcción de saberes y la formulación de preguntas y respuestas.

Todas esas nuevas prácticas del ICACG tienen en común la idea de hacer más transparente, abierta y participativa la construcción de saberes. Esto implica descentrarse del producto, usualmente el texto escrito, desde el que se da cuenta de los resultados de investigación, y visibilizar más el proceso. Construir un puente entre el producto escrito y el proceso que involucra otros artefactos, como bases de datos, entrevistas, repositorios y artefactos digitales de código, implicará nuevas prácticas académicas que pasarán por lo escritural, pero que también necesitan otro tipo de metáforas alrededor de la escritura, que la conecten con todo lo invisible que esta deja atrás. El texto publicado, es entonces solo la "superficie" de la investigación, pero el acto de escribir y publicar dentro y fuera de la academia debe contar con artefactos que den cuenta de sus profundidades y de su carácter no lineal, ya que, además, no vamos del título a las conclusiones de manera organizada, sino que en la medida en que exploramos un problema, se nos ocurren en distintos momentos los elementos que luego incorporamos a esta narrativa lineal y ordenada del texto final.

La ICACG y los objetos de investigación y activistas son parientes cercanos, en el sentido en que consideran maneras alternativas de apropiar, construir y comunicar conocimiento y otros pactos y preguntas posibles en la relación entre ciencia y ciudadanía. A pesar de estar interconectados, entre estos modos de hacer, también existe un dialogo crítico y en ocasiones contrapuesto y no es de extrañar que, al ser un discurso y práctica emergentes, los lugares donde las definiciones y prácticas se consolidan sean principalmente sitios en línea, sin publicaciones canónicas fruto del acuerdo. Se consideran en este apartado algunas definiciones, a fin de dar una mirada panorámica e introductoria al fenómeno ICACG, sin ahondar en los diálogos críticos alrededor del mismo.

Wikipedia (2014) menciona cómo la investigación abierta es cercana a los espíritus del software libre y de código abierto y así como el código fuente se hace disponible en de manera abierta en estos, en la investigación abierta la metodología de investigación se abre, facilitando la colaboración, tanto en el software como en la investigación. Wikipedia también menciona que si bien la investigación puede referirse a hechos factuales de los que se ocupa las ciencias exactas y naturales (llamándose ciencia abierta), la investigación abierta también puede cubrir temas periodísticos, humanistas y médicos, entre otros. La investigación abierta incluye a la ciencia abierta, pero va mucho más allá de los campos científicos y sus formas de narrar. En una derivación de la investigación abierta, llamada investigación reproducible, se pretende que las afirmaciones hechas en la investigación puedan ser contrastados y extendidos por cualquier lector o coinvestigador.

En la experiencia de la comunidad de Grafoscopio, desde sus talleres y hackatones (llamados Data Weeks y Data Rodas), así como en otros encuentros, fue recurrente la presencia de periodistas interesados por el campo del periodismo de datos, bibliotecarios y editores independientes, activistas de derechos humanos en entornos digitales, libertad de expresión, memoria y privacidad, entre otros. Grafoscopio también permite acceder a infraestructura para investigación reproducible, que es de bajo costo y altamente portable y poderosa, útil a todos los perfiles antes mencionados (Luna Cárdenas, 2018, pp.157-191).

Por otra parte, el proyecto del Research Object (s. f.) menciona como los resultados de investigación "no son solo publicaciones tradicionales [sino] todo lo demás que entra en y soporta una investigación”, y menciona cómo el hacer esa diversidad de objetos legibles por máquinas permite que la investigación sea reutilizable, interesante y novedosa, y brinda un conjunto de principios comunes de las distintas iniciativas de objetos de investigación que los haría más ampliamente interoperables y reutilizables. 


\section{El Activist Object (2014) afirma:}

Las infraestructuras digitales, las tecnologías mundanas, las arquitecturas ad-hoc, y los nuevos modos de narrar y documentar están remodelando las prácticas políticas de activistas y ciudadanos. Sabemos que las políticas no solo están hechas de discurso, por el contrario, están hechas de objetos e infraestructuras que deberíamos considerar cuidadosamente. Queremos tomar inspiración de esta idea para aproximarnos a la cultura material del activismo político. Específicamente pretendemos explorar las precarias condiciones del diseño improvisado de los objetos activistas y las implicaciones de las prácticas de documentar y curar los materiales políticos.

La deconstrucción por la que se apuesta en Grafoscopio piensa la documentación como un objeto activista, no solo asociada a las prácticas políticas explícitas, sino aquellas que día a día transitan en los documentos académicos que cosifican la relación poder-conocimiento, pues propone otros artefactos para escribir y publicar dentro y fuera de la academia. También piensa las infraestructuras activistas, pues surge de necesidades sentidas respecto a la creación de capacidad en comunidades de base desde el Hackerspace de Bogotá, HackBo, tanto en sus saberes como en las materialidades que los soportan (Luna Cárdenas, 2018, pp.157-191).

Sobre la ciencia de garaje, Critical Art Emsamble (2009) dice que se trata de un término "rebosante de posibilidades utópicas; sin embargo, a diferencia de otras florituras retóricas utópicas, la forma de producción que describe puede tener un impacto revolucionario en el paisaje de la vida cotidiana”. En ella caben sobre todo las personas cotidianas que están en condiciones de interlocutar con el poder desde los lugares cotidianos (como los garajes), en contraste con la ciencia como aquello exclusivo de los espacios y los dominios cerrados de la academia y los académicos. Por su parte, Lafuente (2014) conecta a la ciencia ciudadana con el movimiento hacker cuando dice que tal movimiento resiste la corporatización del saber, y conecta este movimiento con otros movimientos e iniciativas, como la Wikipedia, los Creative Commons, el movimiento punk.

En resumen, lo que se comparte en las diferentes iniciativas ICACG es la búsqueda de apertura, transparencia y horizontalidad, pero las preguntas, metodologías y artefactos pueden ser muy diversos y con posturas que tienen distintos niveles de diálogo y contrapeso a las dinámicas más hegemónicas de la investigación y la ciencia tradicionales institucionalizadas. Sin embargo, estos artefactos comparten el hecho de estar descentrados del texto y mediados por las tecnologías y representaciones digitales, además de permitir las búsquedas mencionadas.

Grafoscopio se relaciona críticamente con los movimientos de la ciencia de garaje y ciudadana, pues no solo ha ocurrido principalmente en el hackerspace HackBo, sino que desde ahí y en interacción con otras infraestructuras ad hoc, hemos participado en debates sobre asuntos públicos; deconstruimos investigaciones del llamado Big Data, haciéndolas accesibles para muchos o republicamos obras ajenas y propias bajo licencias libres e infraestructura de bolsillo, en contraste con los grandes esfuerzos editoriales que colocan pocas voces en la esfera de lo público (Luna Cárdenas, 2018, pp. 157-191). En todo esto, se explicitan dinámicas e infraestructuras relacionadas con otras maneras de apropiar la tecnología y la ciencia y participar desde dicha apropiación de la vida social y pública y diversificar los sujetos que co-construyen y publican, así como las formas de hacerlo y espacios para ello. Ahí estamos haciendo posible que el ciudadano participe del debate, rete al experto o muestre que esta no es una esfera siempre cerrada y que, por el contrario, puede incluir voces diversas. Estos actos digitales (Isin y Ruppert, 2015) de apertura, clausura, contestación, repolitizan la tecnología y las esferas que ellas habilitan para la co-construcción de lo público.

Desde la comunidad de Grafoscopio, también se piensa la investigación y ciencia ciudadana y de garaje como aquellas que usa los métodos de las ciencias y la investigación para diversificar las voces que participan en ellas, y que se preocupa, particularmente, por la reproductibilidad, verificabilidad y construcción sobre lo dicho, incluso más allá de las prácticas de publicación actuales. En su apuesta por la transparencia como forma de rigor investigativo, en lugar de la supuesta neutralidad o reproductibilidad para todo contexto, trata más bien de tener una reproductibilidad contextual abierta a la reinterpretación constante, facilitada no solo 
gracias al acceso al código fuente, sino a las prácticas educativas comunitarias permanentes, donde este saber se apropia y se cambia.

Lo anterior permite enmarcar el prototipo e indagación abordada por Grafoscopio dentro de la pregunta por un artefacto que, construido desde lo local y considerando las necesidades particulares de lugares específicos en Latinoamérica (el hackerspace HackBo), se está usando para prácticas de ICACG y la exploración y construcción de objetos de investigación y activistas que faciliten los diálogos críticos y los cruces en los discursos y prácticas antes mencionadas.

\section{Principios de diseño de Grafoscopio}

\section{Autorreferencialidad y bootstrapping}

Para construir Grafoscopio se usó una dinámica de bootstrapping (Figura 4), en la cual un sistema mínimo 5 es usado para jalonar instancias más complejas del mismo, que luego remplazan al sistema original. En este caso, para descentrarnos del texto como ejercicio académico por excelencia, se inició escribiendo, de manera emergente y no lineal (siguiendo la jerarquía de clases y métodos de Pharo), en cambio, un artefacto digital para explicitar el carácter no lineal sino arbóreo de la escritura académica, lo que luego permitió escribir el texto desde y sobre dicho artefacto.

Así, la escritura no lineal de código permitió crear un artefacto digital para escritura académica no lineal, que a su vez facilita reflexionar sobre la misma y visibilizar aquellos objetos de investigación que la escritura académica usualmente oculta, incluyendo su propia historia y artefactos conexos, como aquel con el que se inició este proceso. Este artefacto original de escritura es luego extendido en otros contextos y prácticas no académicas (como autopublicación, visualización y narrativas de datos), de manera que va coevolucionando con dichas prácticas y las comunidades y personas en ellas que las desarrollan.

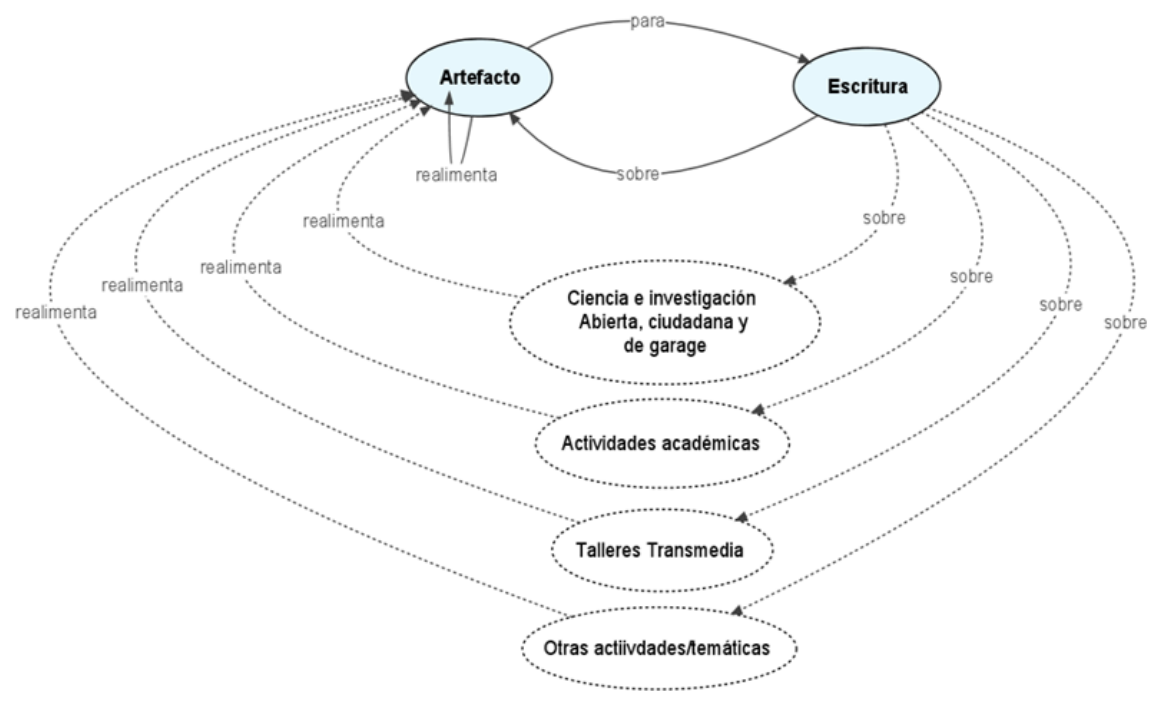

FIGURA 4.

Boostrapping en Grafoscopio.

Nota. Se hizo desde una dinámica de bootstrapping. Se creó un artefacto mínimo para escribir y luego se escribió con él sobre el artefacto mismo. Este ejercicio de escritura, puesto en comunidad. realimentó el (re)diseño y evolución del artefacto. Fuente: Luna Cárdenas (2018).

El Manual de Usuario (Luna Cárdenas, 2017), artículos académicos bocetados y publicados (Luna, 2017a), así como otros documentos PDF, web e interactivos han sido escritos usando Grafoscopio, mostrando las capacidades autorreferenciales y de bootstrapping antes enunciadas: se crea un software mínimo para escribir, 
se usa tanto para escribir sobre el artefacto mismo, como para hacer documentación interactiva sobre otros artefactos y prototiparlos. Con este ejercicio de escritura se realimenta el diseño del artefacto, con lo cual se adapta a procesos de escritura futura más versátiles y potentes, intentando un ciclo virtuoso, anunciado en la Figura 4.

En otras palabras, la idea de autorreferencialidad, de la que se ocupa el diseño (Jonas, 2004), toma cuerpo en este artefacto digital y las prácticas con este, de dos maneras:

- Es un artefacto hecho para escribir, en particular sobre el artefacto mismo, lo cual genera ciclos de realimentación que cambian tanto el artefacto como el proceso de escritura (Figura 4).

- La tecnología principal con la que está hecho Grafoscopio, Pharo, es un metasistema (Denker, 2016), es decir, un sistema tecnológico hecho en sí mismo, con lo cual permite mayor simplicidad, deconstrucción y extensibilidad. A esto se suma la característica de programación en caliente (live coding) que permite alta maleabilidad e interactividad.

Estas dos maneras se combinan en una idea fuerza. Al escribir en Grafoscopio documentos interactivos que requieren el desarrollo de competencias computacionales para modelar y hablar de fenómenos complejos mediados por datos y sus visualizaciones y/o para hacer publicaciones, el autor de tales documentos aprenderá no solo el lenguaje y entorno para su problema/prototipo, sino aquel con el que está hecho todo el sistema. Es decir, en el camino de hablar sobre un fenómeno mediado por simulación, modelación, datos, visualización y/ o publicación, aprenderá a cambiar la herramienta que le permite establecer dicho diálogo. Así, la herramienta que afecta sus maneras de pensar, percibir y expresar un problema, usando documentos interactivos y visualizaciones, puede ser cambiada de vuelta por el autor/lector, de tales documentos y visualizaciones.

Rushkoff (2010) habla de una barrera entre los usuarios y hacedores de artefactos digitales, medida por la programación, que se ilustra particularmente con el software para escribir:

[...] Pero la capacidad subyacente de la era de la computación es de hecho la programación —la cual casi ninguno de nosotros sabe cómo hacer. Simplemente usamos los programas que han sido hechos para nosotros, y entramos nuestro texto en la caja apropiada en la pantalla. Le enseñamos a los niños cómo usar el software para escribir, pero no cómo escribir el software. (p. 13)

En ese sentido, Grafoscopio usa la escritura y publicación de historias soportadas/orientadas por datos para tender un puente entre el "software para escribir" y "escribir el software".

\section{Bifurcación y recombinación}

Desde Jonas (2007), Grafoscopio siguió la estrategia de diseño para nuevos artefactos, que implica el estudio de los puntos de bifurcación de artefactos previos y las posibilidades de diálogo entre tales bifurcaciones, ahora con el beneficio de la retrospectiva histórica. El prototipo busca ser consecuente con las epistemologías del diseño que requieren de nuevos artefactos que permitan explorarlas y comunicarlas y que deberían dar cuenta de sus ingredientes e historia, para mostrar que los "metabolismos cognitivos", como diría Bonsiepe (2000), propios del diseño no son solo anabólicos (de juntura, simplificación y recombinación, que son en los que Bonsiepe se centra), sino, en palabras de Luna Cárdenas (2018), catabólicos (de liberación de energía y componentes para futuras recombinaciones). A continuación, se mencionará como Grafoscopio da cuenta de dichos puentes entre tradiciones y bifurcaciones y de los componentes que permiten la recombinación y el metabolismo cognitivo a partir de los mismos.

Informalmente, se habla de dos "mantras" de la computación en paradigmas distintos, que marcaron puntos de bifurcación a comienzos de la misma. Por un lado, estaba la tradición y el mantra Unix de "todo es un archivo" y la Pharo/Smalltalk ${ }^{6}$ y el mantra de "todo es un objeto". La primera intensión de Grafoscopio era juntar estas dos tradiciones, ofreciendo un modelo en el cual los objetos representan archivos, que 
son documentos arbóreos interactivos para hacer narrativas computacionales. Grafoscopio une estas dos tradiciones al ofrecer herramienta para documentar, simular y visualizar, que son "internas" del entorno Pharo, pero que pueden producir documentos "externos" al mismo y con un público objetivo que no se centra en niños, jóvenes o programadores profesionales (a quienes habitualmente ha estado enfocado Smalltalk), sino que incluye activistas, periodistas, comunicadores, filósofos, investigadores académicos, químicos farmacéuticos, microbiólogos, bibliotecarios, entre otros, considerados a partir de la población que ha asistido a los talleres del Data Week (Figura 5) (Luna Cárdenas, 2018).

Grafoscopio dialoga con ideas de Victor (2014) y Kay (1997 citado en Maxwell, 2006), respecto a medios dinámicos que habiliten formas de pensar un problema de manera multimodal, para entenderlo y expresarlo mejor (Figura 7). Sin embargo, a diferencia de los proyectos de estos autores, no está preocupado con lo que puede ocurrir en el futuro, dentro de 40 años, como el proyecto de Kay en Xerox de los 70 o el Victor de hoy en Dynamicland (Victor et al., 2017), sino con los puentes entre ese futuro y las prácticas presentes. Pues, como la historia ha mostrado, el puente entre el presente donde se exploran aquellas visiones utópicas y el futuro al que pretenden llegar décadas después no es automático y de hecho con frecuencia suele ser ocupado por la distopia. La investigación Tracing the dynabook (Maxwell, 2006) muestra la diferencia entre el mundo que Kay y su equipo buscaban hace décadas y el que tenemos hoy en día, lleno de "usuarios finales", apps para el consumo de contenidos y no para su creación y distante del pensamiento crítico empoderado por el computador. Por ello, la preocupación por el presente y los contextos locales es el foco de Grafoscopio en estos actos de bifurcación y recombinación alineados con la idea de construir un mundo plural y humano, alineándose con las apuestas encarnadas en HackBo. 


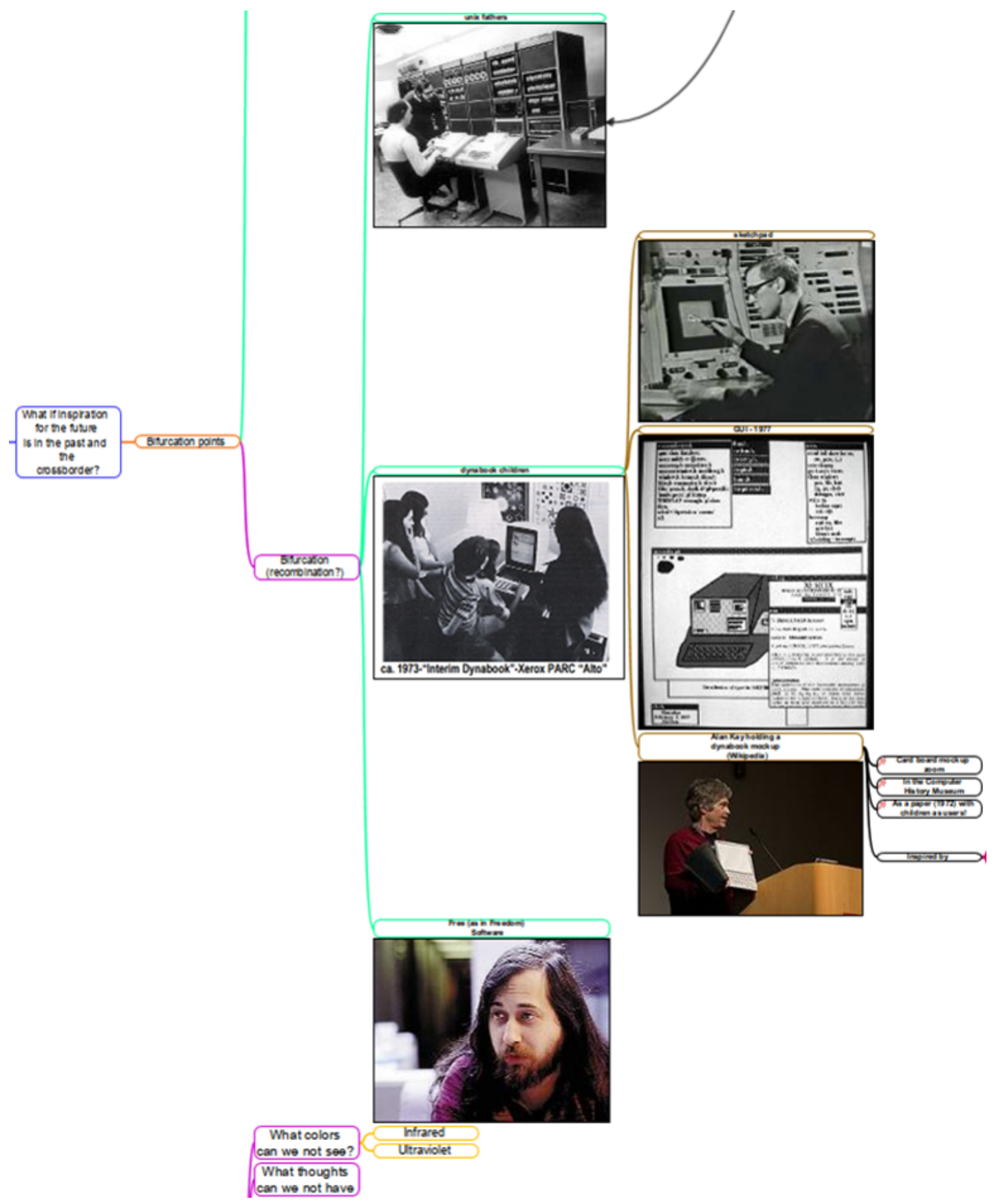

FIGURA 5.

Detalle del mapa mental usado en los Data Weeks.

Nota. Se muestran distintas tradiciones que Grafoscopio recombina y reinterpreta. El mapa completo puede ser visto y descargado en distintos formatos desde Luna Cárdenas (2014). Fuente: Luna Cárdenas (2014). 


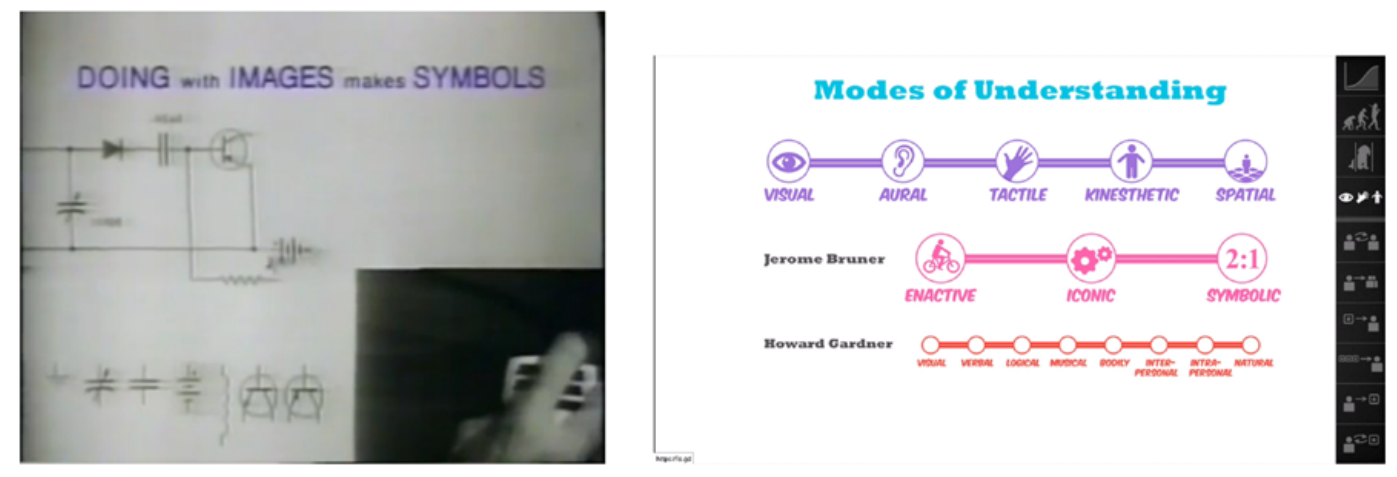

FIGURA 6.

Izquierda: Kay (1997); Derecha: Victor (2014).

Nota. Las figuras aluden a cómo los medios dinámicos, potenciados por tecnologías digitales, pueden favorecer la exploración, comprensión de un mundo de maneras multimodales. Grafoscopio aborda esta comprensión multimodal de una manera particular, al vincular código, texto, datos y visualizaciones para explorar y expresar problemas desde comunidades de base. Fuentes: Kay (1997) y Victor (2014).

Grafoscopio resuena con otras preocupaciones del presente, respecto a narrativas computacionales que toman cuerpo en distintos artefactos, pues al igual que muchos de ellos combina y provee funcionalidades para la prosa y el código con visualizaciones, en libretas y documentos interactivos. Sin embargo, se distancia de estos al desarrollarse en un entorno continuo de cómputo, que no separa en capas disyuntas, lenguaje de programación, Entorno Integrado de Desarrollo (IDE, por sus siglas en inglés), los gestores de código, la aplicación y el documento ${ }^{7}$. Además, usa representaciones simbólicas (código) y gráficas (visualizaciones) para abordar un problema. De esta manera, facilita difuminar la distinción entre usuario y hacedor, problema central para esta investigación y otras como las del Feminismo de Datos (D’Ignazio y Klein, 2020) que cuestionan críticamente los lugares binarios y naturalizados del poder habitualmente embebidos en los artefactos digitales, sus prácticas y sujetos hegemónicos. Otra tradición importante que Grafoscopio recoge es la mirada tecnopolítica del software libre, pues se acoge a una de las licencias que lo cobijan (MIT License, 2020) y explicita, en muchos de los talleres que se hicieron, la idea de la tecnología digital como una manera de hacer viable (o no) el conocimiento como bien común. También expresa las tensiones de estas otras formas de pensar que enfrenta el derecho de autor, pues si bien el código está bajo licencia MIT, su manual (Luna Cárdenas, 2017b) emplea la Licencia de Producción entre Pares (Peer Production License, 2019), que realiza una discriminación positiva para entidades asociativas cooperativas y cooperantes.

Es precisamente en los problemas que se abordan y los prototipos que se crean donde se pueden explicitar estos puentes y tensiones entre tradiciones y bifurcaciones, tratados anteriormente. Luna Cárdenas (2018) detalla varios de los constructos creados con Grafoscopio que cristalizan dichos puentes/tensiones.

\section{Conclusiones}

Grafoscopio es una manera concreta de hablar y encarnar la indagación por la modificación recíproca entre comunidades de base y artefactos digitales, lo cual implica conectarse con las materialidades subyacentes en la tecnología, pero también pensarlas más allá de las ciencias de la computación o una perspectiva positivista del mundo y el código para mirarlas desde discursos críticos en diseño y con implicaciones desde la Comunicación para el Cambio y Desarrollo Social.

Grafoscopio realiza dos exploraciones en paralelo. Por un lado, facilita un diálogo entre tecnología y comunidades de base, para amplificar sus voces, permitiéndoles participar de maneras diferentes de la construcción del discurso público y la comprensión de problemas complejos. Por otro, da claves desde el diseño basado en investigación para facilitar la modificación recíproca entre artefactos digitales y comunidades de base: la autorreferencialidad en el sistema artefacto-comunidad (explorada en las dinámicas 
de escritura) y en el uso de metasistemas; la recombinación de distintas tradiciones informáticas desde el estudio de sus puntos de bifurcación; así como el uso de los ciclos de realimentación, considerados en la metodología del software como hipótesis y como saber artesanal, que implica hacer parte de comunidades preexistentes, conformar nuevas e interconectarlas.

Todo esto permiten diseñar entre, con y para una comunidad de base (en este caso la congregada en HackBo y la comunidad de Grafoscopio), en consonancia con sus apuestas cívicas, y también que las apropiaciones individuales sobre el software y sus materialidades convoque a comunidades más amplias alrededor de las propuestas que cristalizan en ese software. Es decir, acá el software no solo es un prototipo que comunica un saber y apuestas individuales, sino que convoca y permite construir colectivos alrededor de las mismas, para revisarlas y modificar el saber embebido en dicho software.

Se indaga también, en concreto, sobre los metamedios, pues, gracias estar desarrollado en un metasistema de live coding continuo, que diluye las fronteras entre código fuente y aplicación, no solo se embebe un discurso sobre como combinar otros medios (prosa código, datos, consultas) en publicaciones, visualizaciones y narrativas de datos ágiles y personalizadas, sino que también contiene uno sobre cómo el artefacto mismo puede ser apropiado y deconstruido. Sin embargo, hacer plural esta posibilidad es aún un desafío presente, pues al igual que en la mayoría de proyectos de software libre (Eghbal, 2016; Mako, 2013), aún la base de desarrolladores en Grafoscopio es muy pequeña y el código que potencia estas prácticas comunitarias debe ser escrito de maneras aún más colectivas.

Si bien se exploraron puentes vía la publicación académica y comunitaria tanto de artículos académicos como de otros objetos no hegemónicos, queda un desafío sobre cómo estas prácticas en los márgenes pueden ser sostenibles, escalables y visibilizadas en contextos más amplios. Las claves materiales e investigativas, así como las prácticas comunitarias que artefactos como Grafoscopio (de)construyen, son una invitación también a explorar juntos estos desafíos y posibilidades.

\section{Referencias}

Activist Object. (2014). Curating the activist object. About the activist object. http://activistobject.wordpress.com/ab out/

Blackwell, A., y Aaron, S. (2015). Craft practices of live coding language design. Proceedings of the First International Conference on Live Coding (pp. 41-52). Univeristy of Leeds. https://doi.org/10.5281/zenodo.19318

Bonsiepe, G. (mayo, 2000). Design as tool for cognitive metabolism: From knowledge production to knowledge presentation. Trabajo presentado en el International Symposium on the Dimensions of Industrial Design Research, Politecnico di Milano, Italia.

Carretero, A. B. (2019). Comunicación, ciudadanı\#a y cambio social. Diseño de un modelo de investigación y acción para democratizar la comunicación desde la noción de reforma mediática. Signo y Pensamiento, 38(75). https:/ /doi.org/10.11144/javeriana.syp38-75.cccs

Coleman, G. (2013). Coding freedom. The ehics and aesthetics of hacking. Princeton University Press.

Colquhoun, D. (5 de septiembre de 2011). Publish-or-perish: Peer review and the corruption of science. The Guardian. https://www.theguardian.com/science/2011/sep/05/publish-perish-peer-review-science

Critical Art Emsamble. (2009). Ciencia de garaje. https://www.medialab-prado.es/actividades/ciencia-de-garaje

Denker, M. (2016). Perfection \& Feedback Loops or: Why worse is better [Software de computación]. https://www.slid eshare.net/MarcusDenker/perfection-feedback-loops-or-why-worse-is-better-65540840

Derickson, K. D., y Routledge, P. (2015). Resourcing scholar-activism: Collaboration, transformation, and the production of knowledge. The Professional Geographer, 67(1), 1-7. https://doi.org/10.1080/00330124.2014.8 83958

D'Ignazio, C., y Klein, L. F. (2020). Data feminism. The MIT Press. 
Ducasse, S., Cassou, D., y Fabresse, L. (5 de junio de 2020). Pharo Vision [Archivo de video]. https://www.youtube. com/watch?v=I60_3QYBFQ4

Eghbal, N. (2016). Roads and bridges: The unseen labor behind our digital infrastructure (Informe de investigación). Fundación Ford. https://www.fordfoundation.org/work/learning/research-reports/roads-and-bridges-the-uns een-labor-behind-our-digital-infrastructure/

Gruber, J. (2004). Markdown (1.0.1) [Computer software]. https://daringfireball.net/projects/markdown/

HackBo. (s. f.). :| hackbo |: Un hackerspace en Bogotá. https://hackbo.co/

Isin, E. F., y Ruppert, E. (2015). Being digital citizens. Rowman \& Littlefield.

Jonas, W. (2004). Design theories and processes are evolutionary artifacts. En W. Jonas y J. Meyer-Veden (Eds.), Mind the gap. On knowing and not-knowing in design (pp. 177-221). Hauschildt-Verlag.

Jonas, W. (2007). Design research and its meaning to the methodological development of the discipline. En R. Michel (Ed.), Design research now. Board of international research in design (pp. 187-206). Birkhäuser Basel. https://do i.org/10.1007/978-3-7643-8472-2_11

Jonas, W., y Meyer-Veden, J. (Eds.). (2004). Mind the gap. On knowing and not-knowing in design. Hauschildt-Verlag.

Kay, A. (1997, octubre 9). The computer revolution hasn't happened yet [Archivo de video]. https://www.youtube.co $\mathrm{m} /$ watch? $=$ oKg1hTOQXoY

Lafuente, A. (2014, septiembre 22). Amateurs, activistas y hackers: tres formas de estar en la ciencia [Mensaje en un blog]. https://www.diagonalperiodico.net/blogs/fuera-clase/amateurs-activistas-y-hackers-tres-formas-estar-la -ciencia.html

Luna Cárdenas, O. V. (2014). Repositorio de documentación de Grafoscopio. https://mutabit.com/repos.fossil/grafosc opio/info/e0de9ed34000f856

Luna Cárdenas, O. V. (2017a). Grafoscopio: A moldable tool for literate computing and reproducible research. The Journal of Open Source Software, 2(18), 251. https://doi.org/10.21105/joss.00251

Luna Cárdenas, O. V. (2017b). Grafoscopio user manual.Zenodo. https://doi.org/10.5281/ZENODO.1974261

Luna Cárdenas, O. V. (2018). Codiseñar autonomías: artefactos digitales amoldables, hacktivismo y ciudadanias (Tesis doctoral inédita). Universidad de Caldas. https://mutabit.com/repos.fossil/doctorado-offray/

Mako Hill, B. (2013, marzo 23). When free software isn't better [Archivo de video]. https://www.youtube.com/watc $\mathrm{h} ? \mathrm{v}=\mathrm{Er} 1 \mathrm{pM} 9 \mathrm{suxvE}$

Maxwell, J. W. (2006). Tracing the dynabook (Tesis doctoral inédita). University of British Columbia. https://open.l ibrary.ubc.ca/cIRcle/collections/ubctheses/831/items/1.0055157

Research Object. (s. f.). Research object overview. Goals, principles and mechanisms [overview]. University of Manchester. http://www.researchobject.org/overview/

MIT License. (2020, noviembre 28). En Wikipedia. https://en.wikipedia.org/w/index.php?title=MIT_License\&old id $=992306653$

O'Neil, C. (2016). Weapons of math destruction: How big data increases inequality and threatens democracy (1.a ed.). Crown.

Pariser, E. (2012). The filter bubble: How the new personalized web is changing what we read and how we think. Penguin Random House.

Peer Production License. (2019, octubre 18). En Wikipedia. https://wiki.p2pfoundation.net/Peer_Production_Lice nse

Rushkoff, D. (2010). Program or be programmed: Ten commands for a digital age. Soft Skull Press.

Saikaly, F. (2005, marzo). Approaches to design research: Towards the designerly way. Proceedings of the 6th International Conference of the European Academy of Design, Design System Evolution. Hochschule fur kunste Bremen, Germany.

Santos, B. de S. (2018). The end of the cognitive empire: The coming of age of epistemologies of the South. Duke University Press. 
Schrock, A. R. (2014). Education in disguise: Culture of a hacker and maker space. InterActions: UCLA Journal of Education and Information Studies, 10(1). https://escholarship.org/uc/item/0js1n1qg

Schrock, A. R. (2016). Civic hacking as data activism and advocacy: A history from publicity to open government data. New Media \& Society, 18(4), 581-599. https://doi.org/10.1177/1461444816629469

Schrock, A. R. (2018). Hackers and makers are ordinary [Epílogo]. En A. R. Schrock y J. Hunsinger (Eds.), Making our world: The hacker and maker movements in context (pp. 313-317). Peter Lang.

Leinonen, T., Toikkanen, T., y Silfvast, K. (2008). Software as hypothesis: Research-based design methodology. University of Art and Design Helsinki. http://www.slideshare.net/teemul/software-as-hypothesis-researchbased-design-m ethodology-presentation

Manzini, E., y Meroni, A. (2013). Emerging User Demands for Sustainable Oolutions, EMUDE. En Design research now (pp. 157-179). Springer. https://link.springer.com/book/10.1007/978-3-7643-8472-2\#about

Victor, B. (2014, octubre). The humane representation of thought. A trail map for the 21st century [Archivo de video]. https://vimeo.com/115154289

Victor, B., Horowitz, J., Iannini, L., y Rizwan, O. (2017). Dynamicland. https://dynamicland.org/

Wark, M. (2004). A hacker manifesto. Harvard University Press.

Wenger, E. (1999). Communities of practice. Cambridge University Press.

Wunderlich, L. (s. f.). [Conceptual support and design]. Elephant in the Lab. https://elephantinthelab.org/

\section{Notas}

* Artículo de investigación científica. Este artículo es una creación original del autor y no ha sido sometido a ninguna otra publicación hasta el momento. Sus textos son derivados de la tesis doctoral para optar por el título de doctor en Diseño y Creación, que ya fue sometida a evaluación y sustentada públicamente.

1 Aun así, hay decisiones tempranas que aún se encuentran en el software y que deben ser cambiadas desde el conocimiento actual y futuro.

2 Nótese que esta metáfora computacional acá es distinta a la metáfora referida posteriormente para presentar la interfaz gráfica y los modos de escritura, aunque las dos se informan mutuamente, atraviesan distintos lugares de la infraestructura computacional.

3 En ese sentido, Grafoscopio se acoge a la menos popular tradición de los outliners, que precisamente colocan como aspecto central de la interacción con el computador la idea de crear jerarquías emergentes.

4 Para más posturas críticas sobre los artículos y la publicación indexada y sus dinámicas reduccionistas y de extractivismo cognitivo véase Colquhoun (2011), Santos (2018) y (Wunderlich, s. f.) .

5 Los lugares de ese carácter mínimo ya fueron enunciados previamente, particularmente en el apartado del software como artesanía: estructura arbórea evidente, lenguajes de etiquetamiento ligeros para lo escritural, sistemas uniformes y minimalistas para su modificación, no ocultación de los lenguajes de dominio específico.

6 Se usa Pharo y Smalltalk en este contexto, pues creadores del primero reconocen al último como su "ancestro espiritual" (Ducasse et al., 2020), y comparten muchas características.

7 El Manual de Usuario de Grafoscopio muestra en detalle el lugar de este software en medio de las otros similares, las ideas de las cuales se inspira y las apuestas de valor agregado del mismo.

\section{Licencia Creative Commons CC BY 4.0}

Para citar este articulo: Luna Cárdenas, O. (2020). Grafoscopio: un prototipo/hipótesis para indagar sobre cómo cambiamos los artefactos digitales que nos cambian. Signo y Pensamiento, 39(77). https://doi.org/10 $.11144 /$ Javeriana.syp39-77.gpic 